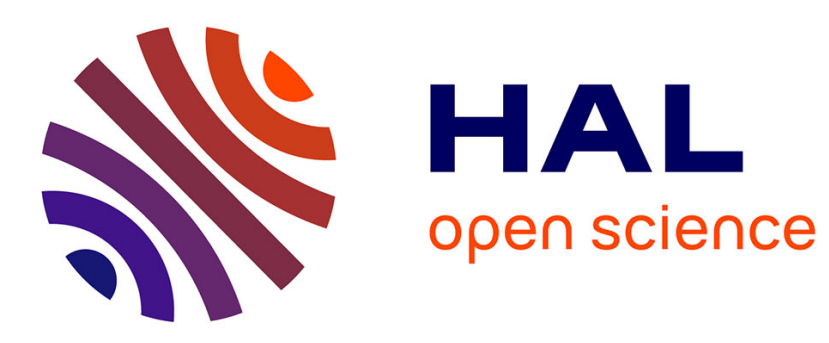

\title{
Video Magnification Applied in Ultrasound
}

\author{
Vincent Perrot, Sébastien Salles, D. Vray, H. Liebgott
}

\section{To cite this version:}

Vincent Perrot, Sébastien Salles, D. Vray, H. Liebgott. Video Magnification Applied in Ultrasound. IEEE Transactions on Biomedical Engineering, 2019, 66 (1), pp.283-288. 10.1109/TBME.2018.2820384 . hal-01894170

\section{HAL Id: hal-01894170 https://hal.science/hal-01894170}

Submitted on 11 Mar 2019

HAL is a multi-disciplinary open access archive for the deposit and dissemination of scientific research documents, whether they are published or not. The documents may come from teaching and research institutions in France or abroad, or from public or private research centers.
L'archive ouverte pluridisciplinaire HAL, est destinée au dépôt et à la diffusion de documents scientifiques de niveau recherche, publiés ou non, émanant des établissements d'enseignement et de recherche français ou étrangers, des laboratoires publics ou privés. 


\title{
Video Magnification Applied in Ultrasound
}

\author{
Vincent Perrot*, Student Member, IEEE, Sébastien Salles, Member, IEEE, Didier Vray, Member, IEEE, \\ Hervé Liebgott, Member, IEEE
}

\begin{abstract}
Goal: This paper describes a method to enhance, visualize and reveal subtle motion that can be present in medical images. As proposed in vision applications, the principle is to magnify displacement applied, in this case, to cardiovascular tissues (carotid). Methods: In the example presented, ultrasound data were acquired at a high frame rate and 2D motion was estimated, amplified and reapplied in ultrasound carotid sequences. Results: Video magnification makes fast and complex phenomena of human tissue visible. In fact, not only pulse and reflected wave, but also global radial and longitudinal motion in the example presented, are visible with video magnification. Conclusion: Video magnification can be used in medical imaging for subtle motion visualization. One of the many possible applications is direct visualization of a local modification in terms of stiffness of a tissue (due to local necrosis, for instance) from acquisition. Moreover, video magnification can be executed with any type of imaging modality. Significance: Video magnification could be a new tool for physicians to highlight new pathology indicators or for long-term disease monitoring.
\end{abstract}

Index Terms-Video magnification, ultrasound, medical imaging, motion estimation, visualization tool.

\section{INTRODUCTION}

$\mathbf{T}$ HE human body and its constituting tissues can be subject to fast, complex and subtle displacements over time. These phenomena are important because they are directly related to the physical properties (geometry and stiffness) of the medium [1], [2] and can be closely linked to their function [3], [4]. For example, the pulse wave velocity of calcified arteries is higher than that of noncalcified arteries. Indeed, such arteries are stiffer and pulse wave velocity is directly proportional to artery stiffness [5]. Furthermore, by modifying the stiffness and the geometry (such as atherosclerosis plaques), the local motion can be reduced in terms of amplitude [5]. Concerning waves, due to the higher velocity induced by the increase in stiffness, reflected and pulse waves will arrive at different times and create new

Manuscript received September 3, 2017; revised February 12, 2018; accepted March 24, 2018.

The Verasonics system was cofounded by the FEDER program, Saint-Etienne Metropole (SME) and Conseil General de la Loire (CG42) within the SonoCardioProtection Project headed by Prof. Pierre Croisille and Dr. Magalie Viallon, principal investigators. This study was also conducted as part of the LABEX CELYA (ANR-10-LABX-0060) and LABEX PRIMES (ANR-10-LABX-0063) projects of the Université de Lyon, within the "Investissements d'Avenir" (ANR-11-IDEX-0007) program operated by the French National Research Agency (ANR). Asterisk indicates corresponding author. Asterisk indicates corresponding author.

*V. Perrot, D. Vray and H. Liebgott are affiliated with the Université de Lyon, INSA Lyon, Université Claude Bernard Lyon 1, Université Jean Monnet Saint-Étienne, CNRS, Inserm, Centre de Recherche en Acquisition et Traitement de l'Image pour la Santé (CREATIS), UMR 5220, U1206, F-69621, Villeurbanne, France (e-mail: vincent.perrot@creatis.insa-lyon.fr).

S. Salles is affiliated with the Department of Circulation and Medical Imaging, Norwegian University of Science and Technology, Trondheim, Norway. motion patterns in the tissue. More generally, modifying the tissue characteristics have a direct impact on motion. To study and understand the motion of tissues in healthy and pathological cases, it is essential to render and represent tissue displacement as accurately as possible. Defining a clear and realistic representation is a difficult task and can be dependent on the application. Typically, some phenomena can be invisible at a specific time and spatial scale and appear completely obvious at another time and scale.

Currently, in standard representation methods, motion maps are mainly displayed by an overlay with the anatomical input data. For example, in these conventional approaches, vectors and colors are frequently used for this purpose [6]. In Fig. 1, the image of a carotid artery is superimposed with velocity vectors along the wall computed between two successive frames of an ultrasound sequence. Some anatomical information is hidden behind the arrows and hardly visible. Vectors and colors partially obscure the image and more specifically the walls, which is an inherent drawback of this type of representation with an overlay. Moreover, with these approaches motion is converted into another format for visualization (colors and vectors in Fig. 1), which implies knowing and explaining the established convention. Furthermore, with vector representation the real size of vectors must be increased to visualize small motion. Consequently, if estimated motion cannot be seen with the naked eye, anatomical information is motionless while vectors, representing motion, move through an apparently immobile medium. This effect might be confusing and gives the impression that anatomical and motion information are dissociated.

On the other hand, a very interesting approach to motion (or other subtle image changes in videos) rendering has been developed for vision applications. The principle of the technique, video magnification, is to extract the relevant subtle information (e.g., motion or small color changes) and increase its scale while applying video magnification to the original images [7]-[9]. For example, in [7], video magnification was used to visualize the breathing of a sleeping newborn and soft wind motion through trees. This technique has, to the best of our knowledge, never been applied directly to ultrasound images to study and represent tissue motion. However, it has been applied with MRI to amplify subtle spatial variations in cardiac-gated brain [10]; the group reused the Eulerian video magnification explained in [7]. In ultrasound, color overlays have been used to visualize the propagation of waves in cardiovascular tissues [11]. Another approach was successfully established for flow visualization, based on speckle enhancement, which also used an amplification process for reporting complex and fast 


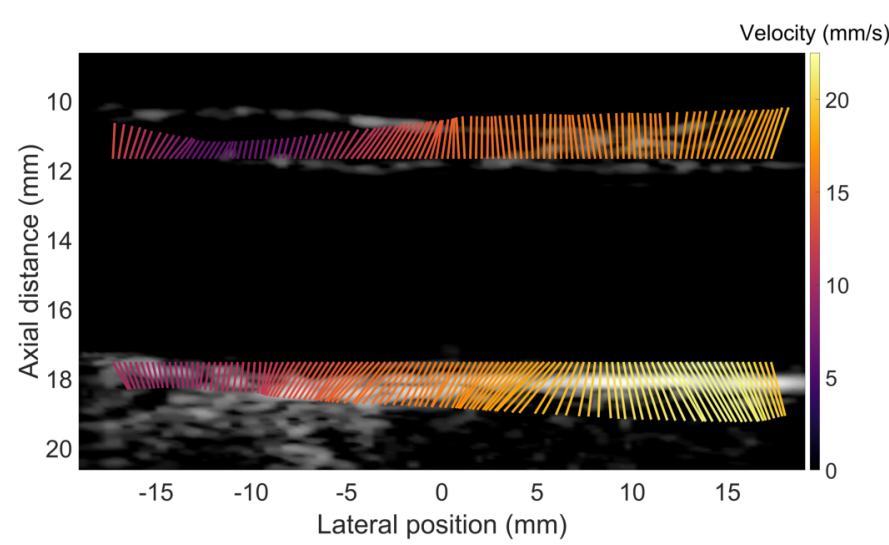

Fig. 1. Example of a motion illustration using color vectors applied to the carotid from ultrasound data. Computation was done between two successive frames (at the frame rate of 2500 images per second). Vectors illustrate motion along the proximal (depth, $11 \mathrm{~mm}$ ) and distal (depth, $17 \mathrm{~mm}$ ) walls; the norm (colored) is proportional to displacement amplitude when direction is connected to motion direction. The image shows a wave (on the proximal and distal walls) with a minimum value (in terms of amplitude) between -15 and $-10 \mathrm{~mm}$ and a maximum value around $10 \mathrm{~mm}$ along the lateral position axis.

phenomena applied to flow [12]. The algorithm represents flow information in a hybrid form comprising a flow speckle pattern and color-encoded velocity mapping flow. Moreover, another group has worked on a tool based on video magnification in a medical context as an aid for isolating a needle from its environment [13]. This method, inspired by Eulerian video magnification [7], is based on the tremor motion of the needle to extract the needle trajectory inside soft tissue.

In this paper, video magnification has been adapted and applied to ultrafast ultrasound data acquired on the common carotid artery aiming to study and highlight complex motion that can occur in the vessel wall. This is only one of the many potential applications in medical imaging that could benefit from video magnification. The research has been focused on the development of a new tool for representing motion on anatomical data. The main objective of this technique is to provide additional motion information without obscuring the original anatomical information.

Section II presents the general method of video magnification broken down into three steps (estimation, magnification and interpolation). Section III describes the application of this technique to an ultrasound example with the experimental set-up and the parameters used for the computation. Section IV presents the results of the method applied to the carotid artery. Section V discusses the tool and the possible applications. Finally, section VI concludes the paper with a brief summary highlighting the significance and the opportunities provided by this tool in biomedical imaging.

\section{GENERAL METHOD}

A simplified, intelligible and efficient approach for video magnification performed in post-acquisition is presented. It is a solution to the problems outlined above, namely the overlay and the format of the visualization. In fact, based on an amplification process of natural motion there is no need to overlay the displacement or convert it to another format

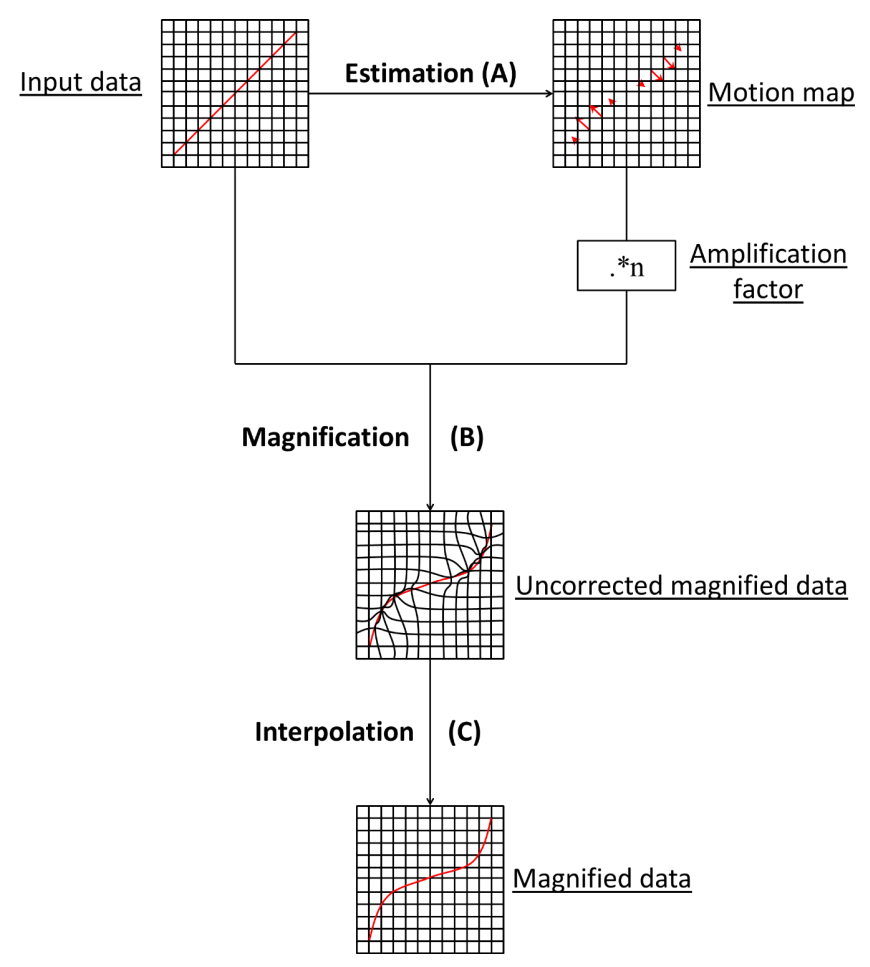

Fig. 2. Simplified block diagram algorithm of the video magnification technique in three steps, based on a basic example to understand the concept of the video magnification algorithm. Bold text is for the stages and underlined text describes each block of the diagram. On the maps, the grid represents the pixel grid, the diagonal line is the object of interest and the vectors indicate motion.

for display. This method is a way to visualize small and subtle displacements that occur in biomedical tissue over time. This tool has been developed as a visualization aid and not for quantification purposes. However, displacement values are extracted during the estimation step (Fig. 2, step A) and can be analyzed quantitatively if necessary. Video magnification could make it possible to easily understand complex and fast tissue phenomena and could also be used for a qualitative inspection or for educational purposes.

Video magnification can be broken down into three major steps after data acquisition:

A. Estimation: measure motion frame by frame for each pixel.

B. Magnification: reapply displacement for each pixel and each frame with an amplification factor.

C. Interpolation: interpolate the (displaced) pixel intensities onto the original rectangular grid.

The algorithm is explained with a simple example through a block diagram in Fig. 2.

The data format (before estimation) depends on the algorithm of the estimation tool. There is no constraint on the format for video magnification; however, the data must have sufficient resolution (spatially and temporally) so as to capture the motion of interest.

The method for motion estimation is linked with the biomedical imaging modality and depends on the user. The displacement estimation technique must provide a velocity value for each pixel and each frame, and a continuous and 
dense Eulerian velocity data field is necessary for video magnification. Motion should be smoothed in both image directions and temporally to obtain an acceptable displayed result; spatio-temporal filtering can be used for this purpose. Therefore, filters are certainly necessary, but the spatial and temporal filters have to be chosen in accordance with the phenomena of interest. An inappropriate filter can remove useful information if the spatial or temporal kernels of the filter are larger than the spatial or temporal variation of the phenomenon studied.

The amplification step is the most important part of the video magnification process. Indeed, the amplification factor must be sufficient to reveal subtle but not excessive displacement; otherwise images will be distorted with a non-natural pattern. The choice of the amplification factor depends on the maximum value of motion and the spatial and temporal data resolution.

Due to the deformation field applied with an amplification factor (the magnification step) the pixel grid is distorted. However, to visualize the new magnified sequence it is necessary to have an orthogonal pixel grid. There are several interpolation methods to regain a corrected grid. Once again, the choice for the interpolation method depends on the imaging modality and the user.

\section{ApPliCATION TO UltRAFAST ULTRASOUND IMAGING OF THE CAROTID ARTERY}

\section{A. Experimental set-up}

In this paper ultrasound data from a previous study, described in [14] were exploited. A Verasonics ultrasound system (Verasonics Inc., Redmond, WA, USA) with the L7-4 probe were used. The acquisition parameters are described in Table I. Acquisitions were performed using plane waves and at a high frame rate of imaging (Table I, 2500 images per second) to avoid aliasing in the estimate by reducing displacement between successive frames. Only one horizontal plane wave was transmitted in order to achieve ultrafast imaging, and the reconstruction was done using Stolt's migration technique [15].

TABLE I

ACQUISITION PARAMETERS

\begin{tabular}{ll}
\hline \hline Parameter & Value \\
\hline Probe & $\mathrm{L} 7-4$ \\
Pitch & $296 \mu \mathrm{m}$ \\
Number of elements & 128 \\
Transmit frequency & $5 \mathrm{MHz}$ \\
Sampling frequency & $25 \mathrm{MHz}$ \\
Transmit pulse & $1-$ cycle sinusoidal pulse \\
Speed of sound & $1540 \mathrm{~m} / \mathrm{s}$ \\
Pulse repetition frequency & $2500 \mathrm{~Hz}$ \\
Compounding & No $($ single plane wave $)$ \\
Steering angle & $90^{\circ}$ \\
Frame rate & 2500 images per second \\
Number of frames & 2500 images $(1 \mathrm{~s})$ \\
Transmit/receive apodization & Rectangular \\
\hline \hline
\end{tabular}

At the time of the medical examination, all subjects were volunteers, healthy, rested and under the age of thirty. These requirements ensure that all phenomena are fully natural and not due to an external criterion or to an internal disorder.

Carotid arteries are subject to fast and complex phenomena during cardiac cycles such as the pulse wave, the reflected wave as well as longitudinal shear stress; these phenomena are difficult to grasp, especially since the amplitude of the motion is relatively reduced compared to the observed scene. Consequently, video magnification appears to be adapted to making a qualitative analysis of the phenomena occurring in the arteries and to emphasize abnormalities.

\section{B. Magnification process}

Data processing was performed using the MATLAB software package (MATLAB R2017a, The MathWorks Inc., Natick, MA, USA).

In the example tested herein, motion was estimated using the transverse oscillation technique [16], [17]. A flow chart diagram is provided in Fig. 3 for understanding the estimation algorithm. This estimation tool was developed for multidimensional motion estimation from high frame rate ultrasound images and is a Eulerian method; consequently, this technique is well suited to the example given in the article. This method consists in multiplying the 2D Fourier spectrum of each ultrasound image by a mask in order to keep only the desired lateral wavelength that will be used for motion estimation (Fig. 4). The resulting Fourier spectrum is composed of four spots corresponding to the axial (natural) and lateral (introduced with mask multiplication) frequencies (Fig. 4). The transverse oscillation was implemented in post-acquisition, in the Fourier domain, with wavelength of $1.8 \mathrm{~mm}$ along the transverse direction (the direction in which an oscillation is created) [18] while natural ultrasound wavelength was $0.3 \mathrm{~mm}$ along the axial direction. Afterwards, analytic signals are extracted using Hann's approach, which consists in extracting two single orthant analytical signals from the original 2D image [19]. Phase over time was estimated on analytic signals through a sliding window measuring 64 pixels per 10 pixels $(2.00 \mathrm{~mm}$ per $2.96 \mathrm{~mm})$ to avoid outliers in the estimate using the least square method. Moreover, a temporal Hamming filter of 25 frames $(10 \mathrm{~ms})$, applied after the phase estimation process, was also imposed to smooth phase estimates for better visualization of the video after the magnification process. Finally, motion was recovered after a multiplication of $1 / 4 \pi f$, as written in (1), on the phase shift [20]:

$$
\left\{\begin{array}{c}
d_{z}=\frac{\Delta \overline{\Phi_{z}}}{4 \pi f_{z}} \\
d_{x}=\frac{\Delta \overline{\Phi_{x}}}{4 \pi f_{x}}
\end{array}\right.
$$

where $z$ and $x$ are the subscripts for the axial (along the ultrasound beam) and the lateral (perpendicular to the ultrasound beam) axes, respectively. $d$ is the estimated displacement for a pixel of the medium at a specific time, $\Delta \bar{\Phi}$ corresponds to the average phase change between two successive frames in the sliding window, and $f$ is the frequency. 


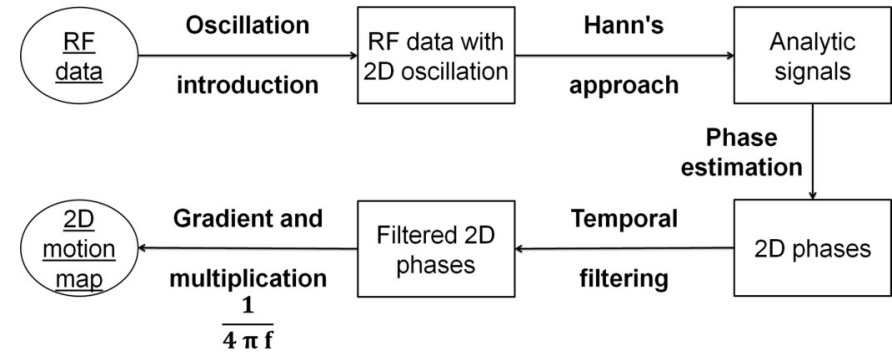

Fig. 3. Flow chart diagram of the estimation algorithm. Bold text stands for the different steps, underlined text corresponds to input data and output results, and boxes are for intermediate outcomes.

Displacement was estimated, according to the video magnification scheme, frame by frame for each pixel, and filtered temporally and spatially (Fig. 2, step A).

With the carotid data, the magnification factor was set between 500 and 1000 (depending on the subject) to obtain approximately $1 \mathrm{~mm}$ maximum magnified motion. This factor was applied globally and provides an amplified but natural motion pattern. For instance, to magnify the pulse wave, the amplification factor was chosen to obtain the resulting maximum displacement amplitude, which corresponds to approximately $10 \%$ of the lumen diameter. This provides easy visualization without a non-natural distortion of the vessel. Then, after the estimation, the selected amplification factor is applied to the pixel position. This requires that the initial pixel position be identified by an orthogonal regular grid: each pixel holds a position (node of the grid) and an amplitude value (intensity). Then the grid is deformed at each node in accordance with the magnified deformation field computed during the previous step. Each pixel is moved to a new set of coordinates corresponding to the amplified motion. The pixel amplitudes remain the same (Fig. 2, step B).

In the example presented, a linear interpolation was used after the magnification step to extract intensities corresponding to the original rectangular grid (Fig. 2, step C). This choice was guided by three causes. Firstly, this type of interpolation is simple to use and establish. Moreover, it is one of the fastest interpolation techniques that allows real-time display. Regarding videos, the method is sufficient and returns results in compliance with estimated motion maps used for the video magnification process. After completing this step, the magnified data are ready for visualization and can be slowly played for understanding and visualizing local, complex and fast motion in the tissue (Fig. 5).

\section{RESUlts}

Once the magnification is completed, the new sequence (magnified) is played slowly, compared with the original frame rate, to appreciate subtle and fast displacement. In this example, the display frame rate is around 60 images per second for an original frame rate of 2500 images per second (approximately 40 times less). Subtle motions are visible with the naked eye ((b) in Fig. 5) with video magnification. This information could not easily be observed on the original data ((a) in Fig. 5).
By viewing the videos ${ }^{1}$ computed with the algorithm, more complex and fast phenomena are also available. Fast phenomena, such as a pulse wave, are visible on the video through the propagation of the wave along the proximal and distal walls. However, it is a fast wave that can exceed $5 \mathrm{~m} / \mathrm{s}$ for a patient [21]. Traditionally, velocity maps or color layered on video are used for this purpose [11], [22], [23]. Concerning complex phenomena, reflected waves, bulk waves (propagation of a longitudinal motion) and local distortion can be studied [14], [23]. In fact, longitudinal motion is related to the depth into the tissue and several layered longitudinal patterns are displayed. Finally, and more generally, all motions (fast, complex, global and local) are directly accessible with this method as amplified information, as long as the motion estimator can measure them.

In terms of computation time, the process can be executed immediately after data acquisition and allows a prompt visualization of the result. As regards the use of this method, there is, for the operator or the person who launches the computation, no special skill required for running the magnification or analyzing and understanding the videos. In fact, all parameters are set inside the code beforehand and the user simply loads or acquires the data and runs the algorithm. Since it can immediately be recalculated, one can imagine that the magnification can be tuned by the user.

\section{Discussion}

This technique is strongly inspired by the Eulerian video magnification proposed in [7], while extending it to medical ultrasound imaging. An adapted estimation method for ultrasound imaging that can extract subtle and fast motion over time based on a phase-based estimator has been exploited. Indeed, the phase from RF signals appears better adapted to extracting subtle displacement in ultrasound with better precision than amplitude [20]. However, because the process is broken down into three different steps and the process is adaptable, the method can be applied to any other motion estimation method or medical imaging modality.

The video magnification technique has several assets. First of all, it can be applied to any biomedical dynamic imaging modality. Motion is not converted but en hanced for visualization, which facilitates understanding and provides a user-friendly result. Last, anatomical information is still present on the final magnified images. However, although the process is easy to run, some parameters must be adjusted and, consequently, several decisions must be made. To begin, the choice of the magnification factor is made by the user and should be in accordance with the phenomena of interest as well as with the imaging modality. In addition, the estimation tool is selected by the user and should be adapted to the data. The robustness of the method is important if one wishes to obtain adequate magnified output. The Eulerian point of view used in the method reduces the risks of divergences due to

\footnotetext{
${ }^{1}$ This paper has supplementary downloadable material available at http://ieeexplore.ieee.org, provided by the authors. This includes three videos illustrating the video magnification (global_longitudinal_motion.mp4, global_radial_motion.mp4, pulsewave_and_reflectedwave.mp4) and a readme file (readme.txt). This material is $1.8 \mathrm{MB}$ in size.
} 


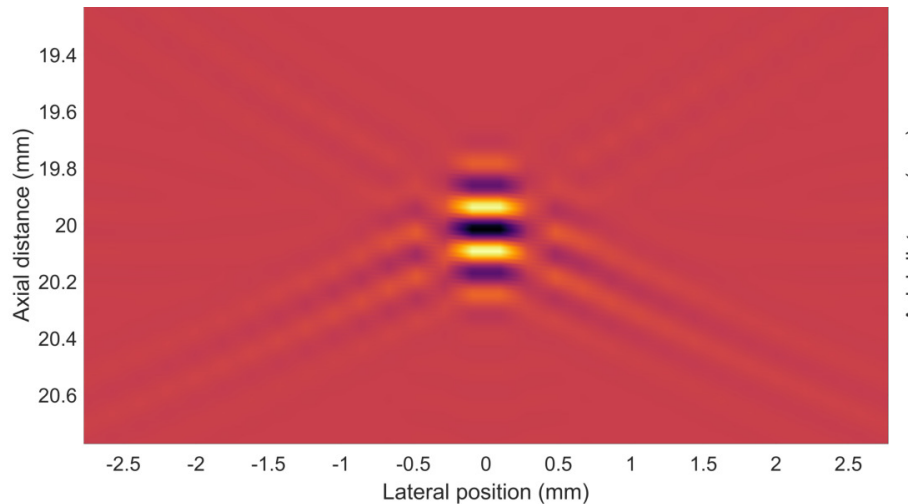

(a)

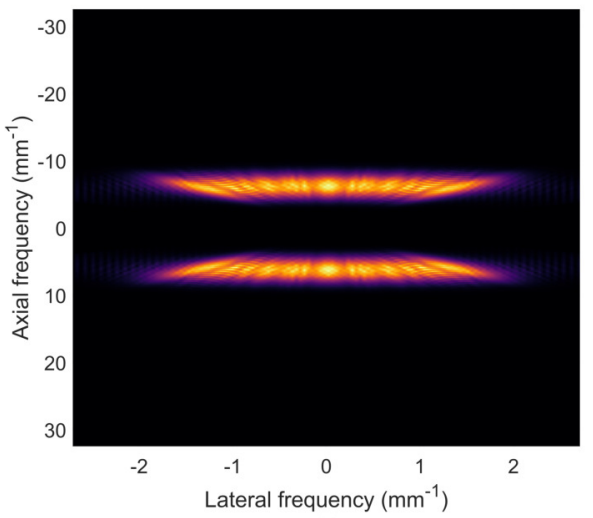

(c)

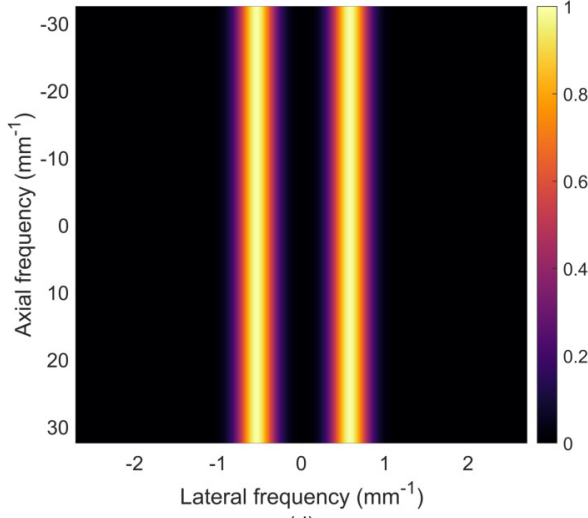

(d)

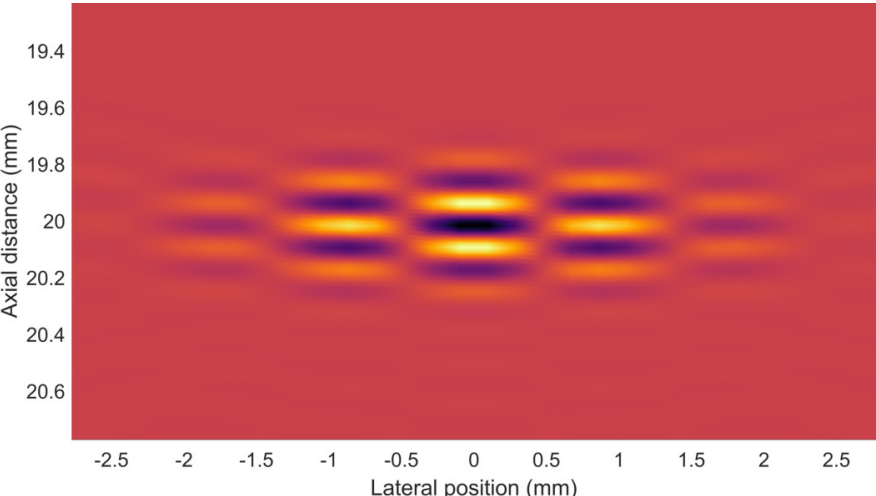

(b)

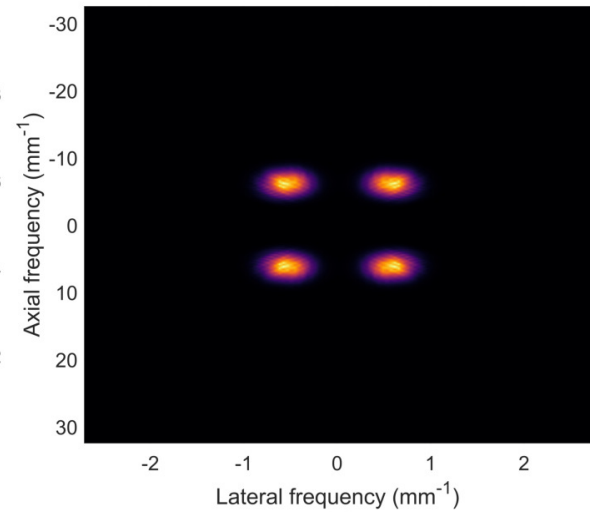

(e)

Fig. 4. (a) Close-up radio-frequency image of a wire. (b) Close-up radio-frequency image with transverse oscillation after filtering. (c) Spatial Fourier transform of (a). (d) Fourier mask for introducing transverse oscillation with a $1.8 \mathrm{~mm}$ wavelength corresponding to a $0.56 \mathrm{~mm}^{-1}$ lateral frequency. (e) Spatial Fourier transform after filtering. Transverse oscillation was introduced (b) in the Fourier domain (e) by applying the mask (d) on the initial Fourier transform (c) of radio-frequency image (a). The resulting radio-frequency image is composed of a $2 \mathrm{D}$ oscillation (b) while only a natural axial oscillation is present in the initial radio-frequency image (a).

error accumulation. Estimation is performed between every two consecutive frames and for each pixel, which means that each estimate is independent of the previous ones, with no error accumulation. Moreover, magnification is also achieved between each pair of frames and with the respective estimated velocity. However, even without error accumultation, some artifacts can be present in images due to the filters if not choosen properly in regard with the imaging sequence and phenomena of interest. Out-of-plane motion can be an issue and distortions can appear if acquisition is not performed correctly. However, this effect can be limited by using a defined and adapted fixed plane of view for medical imaging. For instance, without a 3D imaging technique, a longitudinal cutting plane for carotid arteries or the four-chamber view for cardiac imaging ensure a more stable estimated displacement [24] than other imaging planes but remains challenging in cardiac imaging. In addition, to visualize small displacement in the heart it will be necessary to remove global and large displacement due to the heart beating. For the last step, the correction of the distorted grid (interpolation) also depends on the user and the data (noise, histogram and, more generally, the characteristics of the images).

Concerning the potential usefulness of video magnification, the method could be applied in elastography. This type of tool would indeed be helpful coupled with the transient elastography technique, which induces waves in human tissue [25], [26]. It will be possible to directly visualize the wave propagation and acceleration according to the properties of the medium. Another application could be the amplification of heart waves. In fact, some natural mechanical waves are created by the heart during aortic-valve closure at end-systole [27]. Video magnification offers new perspectives and solutions to visualize stiffness, propagation waves and, more broadly, motion extracted from biomedical images.

\section{CONCLUSION}

In this paper, a video magnification method applied to medical ultrasound imaging has been presented to visualize motion inside human biomedical tissues. The video magnification process has been developed and applied to high frame rate ultrasound imaging of the carotid artery. The technique is divided into three major steps. In addition, the feasibility and utility of the process has been demonstrated. The algorithm is easy to use and offers rapid display after acquisition. Computations were done on a carotid ultrasound acquisition example, but the method is not restricted to this imaging modality. With this tool, global motion, local displacement and distortion as well as complex and fast phenomena within the medium of interest can be seen with the naked eye. There are many possible applications examining 

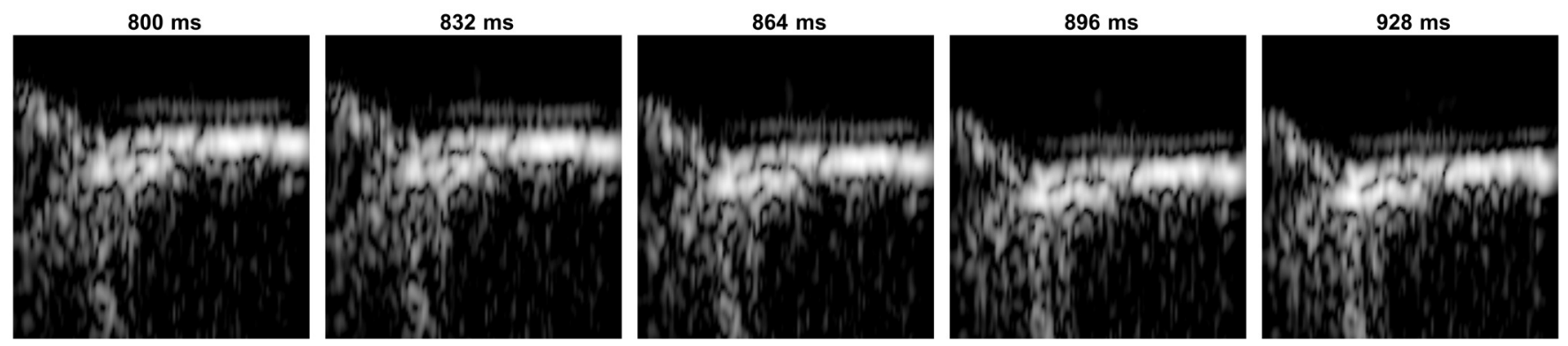

(a)
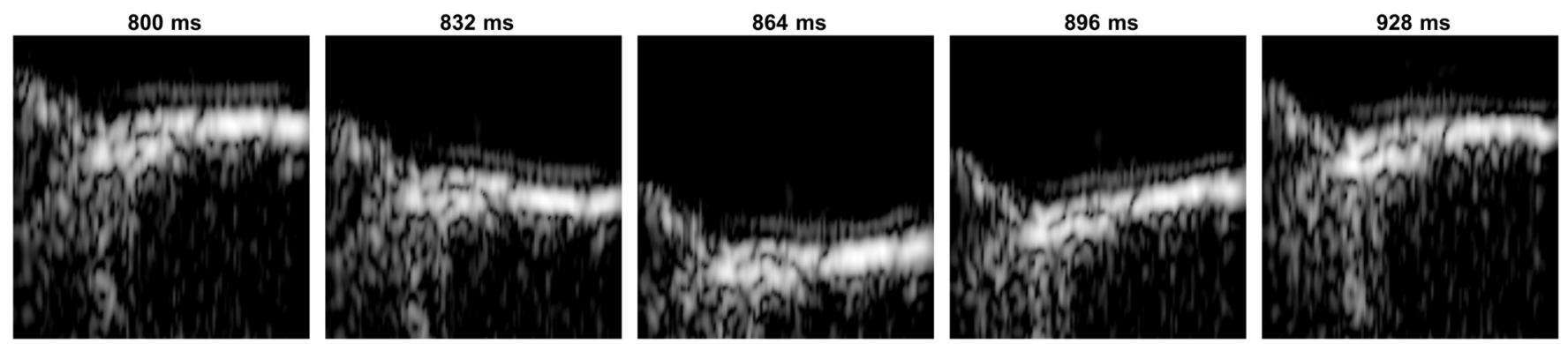

(b)

Fig. 5. Demonstration of the utility of video magnification applied to medical ultrasound imaging for one example with ultrasound (carotid). Close-up views of the distal wall are presented over time $(800-928 \mathrm{~ms}$ after the beginning of acquisition, divided into five images). (a) Input data and (b) magnified data. Radial motion, due to the propagation of a pulse wave, is only visible on the magnified data (b).

the physical properties of tissues. Future research will focus on the implementation of the method to medical devices, made possible by the approaches that were used for the computation. This should allow physicians to employ video magnification in clinical routine as a pre-screening method for diseases or as a visualization tool. In the hands of clinicians, the process could reveal subtle motions, currently not reachable, which might be relevant as pathology markers or for long-term disease monitoring.

\section{REFERENCES}

[1] F. A. Duck, "Mechanical properties of tissue," in Physical Properties of Tissues. Elsevier, 1990, pp. 137-165.

[2] R. Maurice et al., "Noninvasive vascular elastography: Theoretical framework," IEEE Transactions on Medical Imaging, vol. 23, no. 2, pp. $164-180$, feb 2004.

[3] K. Fujikura et al., "A novel noninvasive technique for pulse-wave imaging and characterization of clinically-significant vascular mechanical properties in vivo," Ultrasonic Imaging, vol. 29, no. 3, pp. 137-154, jul 2007.

[4] J. Blacher et al., "Aortic pulse wave velocity as a marker of cardiovascular risk in hypertensive patients," Hypertension, vol. 33, no. 5, pp. 1111-1117, may 1999 .

[5] W. Nichols, M. O'Rourke, and C. Vlachopoulos, McDonald's Blood Flow in Arteries 6th Edition: Theoretical, Experimental and Clinical Principles, R. Reneman, Ed. CRC Press, jul 2011.

[6] D. Laidlaw et al., "Comparing 2D vector field visualization methods: A user study," IEEE Transactions on Visualization and Computer Graphics, vol. 11, no. 01, pp. 59-70, jan 2005.

[7] H.-Y. Wu et al., "Eulerian video magnification for revealing subtle changes in the world," ACM Transactions on Graphics, vol. 31, no. 4, pp. 1-8, jul 2012.

[8] M. Rubinstein et al., "Revealing invisible changes in the world," Science, vol. 339, no. 6119 , pp. 519-519, feb 2013.
[9] M. A. Elgharib et al., "Video magnification in presence of large motions," in 2015 IEEE Conference on Computer Vision and Pattern Recognition (CVPR). Boston, MA, USA, jun 2015.

[10] S. J. Holdsworth et al., "Amplified magnetic resonance imaging (aMRI)," Magnetic Resonance in Medicine, vol. 75, no. 6, pp. 2245-2254, feb 2016.

[11] M. Pernot et al., "ECG-gated,mechanical and electromechanical wave imaging of cardiovascular tissues in vivo," Ultrasound in Medicine \& Biology, vol. 33, no. 7, pp. 1075-1085, jul 2007.

[12] B. Y. Yiu and A. C. Yu, "High-frame-rate ultrasound color-encoded speckle imaging of complex flow dynamics," Ultrasound in Medicine \& Biology, vol. 39, no. 6, pp. 1015-1025, jun 2013.

[13] P. Beigi et al., "Automatic detection of a hand-held needle in ultrasound via phased-based analysis of the tremor motion," in SPIE Medical Imaging 2016: Image-Guided Procedures,Robotic Interventions, and Modeling. San Diego, CA, USA, mar 2016.

[14] S. Salles et al., "Estimation of arterial wall motion using ultrafast imaging and transverse oscillations: in vivo study," in 2016 IEEE International Ultrasonics Symposium (IUS). Tours, France, sep 2016.

[15] D. Garcia et al., "Stolt's f-k migration for plane wave ultrasound imaging," IEEE Transactions on Ultrasonics,Ferroelectrics, and Frequency Control, vol. 60, no. 9, pp. 1853-1867, sep 2013.

[16] J. Jensen and P. Munk, "A new method for estimation of velocity vectors," IEEE Transactions on Ultrasonics,Ferroelectrics and Frequency Control, vol. 45, no. 3, pp. 837-851, may 1998.

[17] H. Liebgott et al., "Transverse oscillations for tissue motion estimation," Ultrasonics, vol. 50, no. 6, pp. 548-555, may 2010.

[18] S. Salles et al., "Plane wave transverse oscillation (PWTO): An ultra-fast transverse oscillation imaging mode performed in the fourier domain for 2D motion estimation of the carotid artery," in 2014 IEEE 11th International Symposium on Biomedical Imaging (ISBI). Beijing, China, apr 2014.

[19] T. Bulow and G. Sommer, "Hypercomplex signals-a novel extension of the analytic signal to the multidimensional case," IEEE Transactions on Signal Processing, vol. 49, no. 11, pp. 2844-2852, nov 2001.

[20] A. Basarab et al., "Phase-based block matching applied to motion estimation with unconventional beamforming strategies," IEEE 
Transactions on Ultrasonics,Ferroelectrics and Frequency Control, vol. 56, no. 5, pp. 945-957, may 2009.

[21] F. U. Mattace-Raso et al., "Determinants of pulse wave velocity in healthy people and in the presence of cardiovascular risk factors: establishing normal and reference values," European Heart Journal, vol. 31, no. 19, pp. 2338-2350, jun 2010.

[22] J. Vappou, J. Luo, and E. E. Konofagou, "Pulse wave imaging for noninvasive and quantitative measurement of arterial stiffness in vivo," American Journal of Hypertension, vol. 23, no. 4, pp. 393-398, apr 2010.

[23] R. X. Li et al., "Pulse wave imaging (PWI) and arterial stiffness measurement of the human carotid artery: An in vivo feasibility study," in 2011 IEEE International Ultrasonics Symposium (IUS). Orlando, FL, USA, oct 2011.

[24] L. G. Rudski et al., "Guidelines for the echocardiographic assessment of the right heart in adults: A report from the american society of echocardiography," Journal of the American Society of Echocardiography, vol. 23, no. 7, pp. 685-713, jul 2010.

[25] J.-L. Gennisson et al., "Transient elastography in anisotropic medium: Application to the measurement of slow and fast shear wave speeds in muscles," The Journal of the Acoustical Society of America, vol. 114, no. 1, pp. 536-541, jul 2003.

[26] L. Sandrin et al., "Fibroscan ${ }^{\circledR}$ in hepatology: a clinically-validated tool using vibration-controlled transient elastography," in 2009 IEEE International Ultrasonics Symposium (IUS). Roma, Italy, sep 2009.

[27] H. Kanai, "Propagation of spontaneously actuated pulsive vibration in human heart wall and in vivo viscoelasticity estimation," IEEE Transactions on Ultrasonics,Ferroelectrics and Frequency Control, vol. 52, no. 11, pp. 1931-1942, nov 2005. 